\title{
Prognostic Factors for Disability and Pain Outcomes in Patients with Axial Low Back Pain Undergoing a Multidisciplinary Spine Treatment Program
}

\author{
Nicholas K Donohue ${ }^{1 *}$, Emily J Graf ${ }^{1}$, Alexis M Visotcky ${ }^{2}$, Sergey S Tarima ${ }^{2}$ and Andrew C Hsu ${ }^{1}$ \\ ${ }^{1}$ Department of Physical Medicine \& Rehabilitation, Medical College of Wisconsin, USA \\ ${ }^{2}$ Division of Biostatistics, Medical College of Wisconsin, USA
}

*Corresponding author: Nicholas K Donohue, MD, Department of Physical Medicine \& Rehabilitation, Medical College of Wisconsin, USA, Tel: 414-805-7342

\begin{abstract}
Introduction: Several studies in the past have examined factors impacting improvement in low back pain in the primary care context. However, there is limited evidence regarding prognostic factors for pain and disability scores in patients with axial low back pain entering a multidisciplinary spine program.

Objective: The goal of this study was to assess which baseline demographic, disability, and pain-related characteristics may be prognostic for improvement in pain and disability scores entering a multidisciplinary spine program.

Design: This was a retrospective review of patients with axial low back pain who participated in a multidisciplinary spine program. Baseline demographic, pain, and disability data were collected and compared to follow-up questionnaire data gathered at discharge. Primary outcomes included change in pain as identified by the Numeric Pain Rating Scale (NPRS) and change in disability as measured by the Oswestry Disability Index (ODI).
\end{abstract}

Setting: A multidisciplinary spine center at an academic institution.

Results: A total of 204 subjects were included in this study. A univariate analysis showed significantly greater improvement in disability scores in the acute/subacute group compared to the chronic group $(p=0.003)$. Subjects with a history of mental health diagnosis experienced significantly less improvement in pain scores $(p=0.042)$. A multivariate regression analysis showed a chronic symptom duration and a higher initial disability score were significant predictors for higher disability scores following completion of the program ( $p=0.003$ and $p<0.0001$, respectively). A mental health diagnosis $(p=0.008)$ and a higher initial pain score $(p<0.0001)$ were associated with less improvement in pain levels, while a higher BMI predicted lower final pain scores $(p=0.020)$
Conclusion: Patients with axial low back pain entering a spine treatment program with higher initial disability and pain scores, a mental health diagnosis, and chronic symptoms may demonstrate less improvement upon discharge.

\section{Keywords}

Axial low back pain, Disability, Multidisciplinary spine program

\section{Introduction}

Low back pain is a common ailment in our society with a prevalence reported as high as $15-20 \%$ and recurrence of pain up to $50 \%$ within one year of onset [1]. This places a significant financial burden on healthcare systems, which has been examined by several previous studies. For instance, Mehra, et al. used a large US commercial insurance claims database to conclude that patients with chronic low back pain without a neuropathic component was found to have a $\$ 1,007$ yearly average cost of care per patient [2]. According to a meta-analysis done by Dagenais $S$, et al. the direct cost of low back pain was found to be $\$ 12.2-\$ 90.6$ billion with indirect costs ranging \$7.4-\$28.2 billion in the US [3]. Given the high prevalence and cost of this illness, there is urgency in identifying factors that hinder recovery or management of low back pain.

Various demographic, disability, and pain-related factors have been studied in the primary care context to investigate their prognostic value for recovery from axial low back pain. Many of these studies have suggested 
that patients with acute or subacute episodes of axial low back pain ( $<3$ months) have greater improvement in pain and disability scores compared to those with chronic symptoms (> 3 months) [4-7]. A mental health diagnosis, specifically a history of depression, has been associated with poorer outcomes in patients with low back pain [8-10]. Increased body mass index (BMI), has also been studied in the context of physical therapy practices and has been associated with decreased response to treatment $[11,12]$. To our knowledge, few studies addressing prognostic factors for improvement have been conducted in the context of a multidisciplinary spine center that incorporates physical and occupational therapy, chiropractic treatment, and interventional spine procedures provided by physiatrists.

The primary objective of this study was to investigate the prognostic value of initial demographic, disability, and pain-related factors on disability and pain scores following completion of a multidisciplinary spine treatment program. Primary outcome measures were changes in the Oswestry Disability Index (ODI) score and Numeric Pain Rating Scale (NPRS) from the initial evaluation to discharge. The ODI and NPRS have been validated in various studies [13-17], and for both the ODI and NPRS, a lower score indicates less disability and pain. A secondary investigation of this study was to determine if there is a correlation between duration of symptoms or a mental health diagnosis and the utilization of the multidisciplinary treatments made available to the subjects, including physical therapy, chiropractic treatment, and interventional spine procedures. We hypothesized that patients with acute/subacute symptoms would have greater improvement in outcomes upon discharge from the program compared to patients with chronic symptoms. In addition, we hypothesized that patients with a longer duration of symptoms or a history of a mental health diagnosis would have increased utilization of treatments.

\section{Methods}

This study obtained approval from the local Institutional Review Board, which provided a waiver of informed consent due to the retrospective design of this study. A retrospective chart review was performed for the time frame of January, 2013, through December, 2015, using billing and coding resources. The following diagnoses were used in the search: ICD9 or ICD10 codes for lumbago, discogenic low back pain, mechanical low back pain (724.2; M54.5), lumbosacral spondylosis without myelopathy (721.3, M47.817 and M47.816), and lumbar facet joint pain (719.48; M54.5). The specific clinical information obtained included basic demographics, duration of symptoms, work status at time of initial visit, smoking status, mental health diagnoses, specific treatment visits for physical therapy, chiropractic visits, lumbar spine injections, duration of treatment, and initial and final

\section{NPRS and ODI scores.}

The inclusion criteria were age $\geq 18$ years and $<65$ years and one of the spine-related diagnoses mentioned above. Exclusion criteria were age $>65$ years to exclude Medicare payers who do not have chiropractic benefits, prior lumbar spine surgery, radiculopathy or symptoms of "sciatica," lumbar spinal stenosis with neurogenic claudication, pregnancy, and those receiving any portion of their treatments from an outside provider. Pregnant women were also excluded from the study due to the possible confounders of pregnancy-related, and therefore time-specific, pain as well as limitations on medications.

\section{Statistical Analysis}

The sample size was conservatively estimated based on the size needed to detect clinically meaningful differences between changes of acute/subacute and chronic pain groups. The Bonferroni adjustment was used for two tests and lowered the ALPHA to 0.0125 . To detect a difference of NPRS $=2$ (conservative $S D=2.5$ ), 37 subjects per group was needed (ALPHA $=0.0125$, POWER $=0.8$ ). . To detect a difference of ODI $=12.7$ (conservative $S D=26), 96$ subjects per group was needed (ALPHA $=0.0125$, POWER $=0.8$ ). Paired t-tests were used to compare pre- and post-treatment outcomes for NPRS and ODI scores. Demographic data and baseline characteristics were analyzed with the following: Continuous variables were compared using Spearman correlations, and categorical variables were analyzed using Wilcoxon-Mann Whitney tests for two groups and Kruskal-Wallis tests for more than two groups. Paired t-tests were used to compare duration of treatment and quantitative utilization of treatments for the secondary investigation.

\section{Results}

A total of 204 patients were included in this retrospective review, and the general demographic information is presented in Table 1. There were 113 females and 91 males, and the average age was 43.2 years (SD 12.9). The number of patients with acute/ sub-acute symptoms was $58(28 \%)$ compared to the chronic group (72\%). A majority of subjects $(76.4 \%)$ had no history of a mental health diagnosis, and $23.7 \%$ had a history of either depression, generalized anxiety disorder, panic disorder, bipolar disorder or a combination thereof. There was no significant difference with regard to mental health diagnosis between the acute/subacute and chronic duration groups ( $p$ $=0.179$ ). The mean initial NPRS and ODI scores for the acute/subacute cohort were 5.0 and 35.3 , respectively. The mean initial NPRS and ODI scores for the chronic cohort were 4.6 and 28.3, respectively. There was no significant difference in initial NPRS scores between the two groups ( $p=0.135)$, but the acute/ subacute cohort did have a significantly higher ODI ( $p$ 
Table 1: Baseline Demographics, Disability Scores, and Pain Scores.

\begin{tabular}{|c|c|c|c|c|}
\hline Variables & $\begin{array}{l}\text { Total } \\
N=204(\mathrm{col} \%)\end{array}$ & $\begin{array}{l}\text { Acute/Subacute Group } \\
\mathrm{N}=58(\mathrm{col} \%)\end{array}$ & $\begin{array}{l}\text { Chronic Group } \\
N=146(\mathrm{col} \%)\end{array}$ & P Value \\
\hline Age & & & & $0.924^{w}$ \\
\hline $\mathrm{N}$ & 204 & 58 & 146 & \\
\hline Mean \pm SD & $43.2 \pm 12.9$ & $43.3 \pm 13.4$ & $43.2 \pm 12.8$ & \\
\hline Gender & & & & $0.968^{c}$ \\
\hline Male & $91(44.6)$ & $26(44.8)$ & $65(44.5)$ & \\
\hline Female & $113(55.4)$ & $32(55.2)$ & $81(55.5)$ & \\
\hline BMI & & & & $0.507^{w}$ \\
\hline $\mathrm{N}$ & 199 & 56 & 143 & \\
\hline Mean \pm SD & $29.7 \pm 7.5$ & $29.9 \pm 7.0$ & $29.7 \pm 7.7$ & \\
\hline Smoking & & & & $0.034^{c}$ \\
\hline No & $186(91.2)$ & $49(84.5)$ & $137(93.8)$ & \\
\hline Yes & $18(8.8)$ & $9(15.5)$ & $9(6.2)$ & \\
\hline Employment Status & & & & $0.641^{c}$ \\
\hline Unemployed & $40(19.6)$ & $9(15.5)$ & $31(21.2)$ & \\
\hline At work & $136(66.7)$ & $41(70.7)$ & $95(65.1)$ & \\
\hline Not working because of pain & $28(13.7)$ & $8(13.8)$ & $20(13.7)$ & \\
\hline Duration of Treatment (weeks) & & & & $0.424^{w}$ \\
\hline $\mathrm{N}$ & 199 & 57 & 142 & \\
\hline Mean \pm SD & $5.9 \pm 5.2$ & $5.5 \pm 5.0$ & $6.1 \pm 5.2$ & \\
\hline Any Mental Health Diagnosis & & & & $0.917^{c}$ \\
\hline No & $155(76.4)$ & $44(75.9)$ & $111(76.6)$ & \\
\hline Yes & $48(23.6)$ & $14(24.1)$ & $34(23.4)$ & \\
\hline Initial NPRS Score & & & & $0.135^{w}$ \\
\hline $\mathrm{N}$ & 204 & 58 & 146 & \\
\hline Mean \pm SD & $4.7 \pm 2.0$ & $5.0 \pm 2.1$ & $4.6 \pm 1.9$ & \\
\hline Initial ODI Score & & & & $0.006^{w}$ \\
\hline $\mathrm{N}$ & 204 & 58 & 146 & \\
\hline Mean \pm SD & $30.3 \pm 17.7$ & $35.3 \pm 17.9$ & $28.3 \pm 17.3$ & \\
\hline
\end{tabular}

$=0.006)$. Initial data were missing for the following categories: 1 subject for mental health diagnosis and 5 patients for BMI.

Table 2 summarizes the unadjusted associations between measured variables and the primary outcome measures. The acute/subacute group on average experienced a significantly greater improvement in ODI (-19.45) when compared with the chronic group $(-9.0)(p=0.003)$. Otherwise there were no statistically significant prognostic factors regarding change in ODI scores. There was no significant difference in change in NPRS scores between the acute/ subacute and chronic groups (0.177). Patients with a history of a mental health diagnosis (NPRS change of -1.71) experienced less improvement in reported pain levels compared to those who did not $(-2.56)$ $(p=0.042)$. Current smokers also experienced a significantly greater decrease in pain levels compared to non-smokers $(p=0.016)$. However, the smoking group was very small $(n=18)$ and smoking status was not significant in the subsequent multivariate analyses.

Two multivariate regression models were conducted to find predictors of change in ODI and NPRS scores while adjusting for the acute/subacute and chronic groups, and the results are represented in Table 3. All variables were considered from the initial univariate analysis but only those found to be significant are presented. The regression analysis for change in ODI showed a chronic symptom duration ( $>3$ months) and a higher initial disability score were significant predictors for higher (worse) disability scores following completion of the program ( $p=0.003$ and $p<0.0001$, respectively). The multivariate regression analysis for change in pain score showed a mental health diagnosis $(p=0.008)$ and a higher initial pain score $(p<0.0001)$ were significantly prognostic for higher pain scores upon completing the program. That analysis also showed that a higher BMI 
Table 2: Univariate Analysis for Change in ODI and NPRS Scores.

\begin{tabular}{|c|c|c|c|c|}
\hline \multirow[b]{2}{*}{ Variables } & \multicolumn{3}{|l|}{ Outcome } & \multirow[b]{2}{*}{$P$ value } \\
\hline & Change in ODI & $P$ value & Change in Middle NPRS & \\
\hline Any Mental Health Diagnosis & & $0.833^{w}$ & & $0.042^{w}$ \\
\hline No & $-11.67 \pm 14.32$ & & $-2.56 \pm 2.17$ & \\
\hline Yes & $-11.95 \pm 15.19$ & & $-1.71 \pm 1.86$ & \\
\hline \multicolumn{5}{|l|}{ Age } \\
\hline Spearman Correlation & -0.079 & $0.366^{s}$ & -0.039 & $0.608^{s}$ \\
\hline \multicolumn{5}{|l|}{ Gender } \\
\hline Male & $-13.84 \pm 14.97$ & $0.229 w$ & $-2.47 \pm 2.09$ & $0.343^{w}$ \\
\hline Female & $-9.69 \pm 13.74$ & & $-2.28 \pm 2.17$ & \\
\hline \multicolumn{5}{|l|}{ BMI } \\
\hline Spearman Correlation & -0.116 & $0.183^{s}$ & 0.004 & $0.95^{\mathrm{s}}$ \\
\hline \multicolumn{5}{|l|}{ Smoking } \\
\hline No & $-11.02 \pm 13.58$ & $0.109 w$ & $-2.27 \pm 2.08$ & $0.016^{w}$ \\
\hline Yes & $-27.0 \pm 24.02$ & & $-4.22 \pm 2.24$ & \\
\hline \multicolumn{5}{|l|}{ Employment Status } \\
\hline Unemployed & $-10.19 \pm 10.87$ & $0.884^{\mathrm{K}}$ & $-2.27 \pm 1.87$ & $0.902^{\mathrm{K}}$ \\
\hline At work & $-11.65 \pm 15.1$ & & $-2.37 \pm 2.15$ & \\
\hline Not working because of pain & $-14.11 \pm 15.45$ & & $-2.46 \pm 2.4$ & \\
\hline \multicolumn{5}{|l|}{ Duration of Treatment } \\
\hline Spearman Correlation & 0.103 & $0.240^{s}$ & -0.123 & $0.109^{s}$ \\
\hline \multicolumn{5}{|l|}{ Group } \\
\hline Acute & $-19.45 \pm 19.18$ & $0.003 \mathrm{w}$ & $-2.73 \pm 2.21$ & $0.177^{w}$ \\
\hline Chronic & $-9.0 \pm 11.28$ & & $-2.22 \pm 2.09$ & \\
\hline
\end{tabular}

Table 3: Multivariate Regression Analyses for Change in ODI and Pain Scores.

\begin{tabular}{|c|c|c|c|c|}
\hline \multirow[b]{2}{*}{ Variables } & \multicolumn{3}{|l|}{ Outcome } & \multirow[b]{2}{*}{ P value } \\
\hline & Change in ODI Estimate (SE) & $P$ value & Change in NPRS Estimate (SE) & \\
\hline \multicolumn{5}{|l|}{ Group } \\
\hline Chronic vs. Acute & $7.37(2.5)$ & 0.003 & $0.276(0.32)$ & 0.388 \\
\hline Baseline Measure & $-0.395(0.1)$ & $<0.0001$ & $-0.574(0.08)$ & $<0.0001$ \\
\hline \multicolumn{5}{|l|}{ Mental Diagnosis } \\
\hline Yes vs. No & & & $0.914(0.34)$ & 0.008 \\
\hline BMI & & & $0.046(0.02)$ & 0.020 \\
\hline
\end{tabular}

was correlated with greater improvement in pain scores $(p=0.002)$. Table 4 summarizes the findings regarding the secondary outcome measures. There were no significant differences in treatment duration or utilization of any of the provided treatments available in the multidisciplinary spine center between the acute/subacute or chronic cohorts nor between subjects with a history of mental health diagnosis and those without.

\section{Discussion}

This study sought to investigate which baseline patient characteristics and presenting disability and pain scores may be significant prognostic factors for pain and disability outcomes for patients with axial low back pain entering a multidisciplinary spine treat- ment program. The main findings of this study show that symptom duration less than 3 months (acute/ subacute) significantly correlated with greater improvement in disability scores while a higher initial disability score was associated with less improvement in ODI. A mental health diagnosis and higher initial pain scores were associated with less improvement in final pain scores, and a higher BMI was associated with greater improvement in pain scores.

Several studies have previously investigated prognostic factors that impact intensity and duration of pain and disability in patients with axial low back pain in the primary care context. A systematic review performed by Valentin, et al. included 19 studies which 
assessed various potential prognostic factors related to duration of disability [5]. Five of these studies found higher initial levels of disability to predict persistent disability, which is consistent with our findings. Two of those studies, however, found no significant association with greater disability. Three of the studies in that review found an association between longer duration of symptoms and prolonged disability, which is consistent with this study. However, definitions for pain duration were not provided in 2 of the studies.

Oliveira, et al. performed a similar study to determine prognostic factors impacting disability and pain in 616 patients with axial low back pain in the context of 4 weeks of physical therapy [18]. Similar to our study, their multivariate regression analysis showed that the baseline pain score was a significant factor impacting final pain scores $(p=0.000)$. In contrast to our study, age was a significant prognostic factor for pain scores $(p=0.003)$ while BMI was not. Regarding disability scores after 4 weeks, they also found baseline disability score $(p=0.000)$ and age $(p$ $=0.001$ ) to be significant prognostic factors. They did not find duration of symptoms to impact either pain or disability levels at discharge, whereas in our study, the acute/subacute pain cohort had significantly improved disability scores after treatment compared to the chronic pain cohort. Also of note, this study did not address any potential mental health factors.

Grotle, et al. performed a similar study in the primary care context to assess whether prognostic factors for axial low back pain were different between patients with acute/subacute symptoms ( $>3$ months) versus those with chronic symptoms $(n=258)$ [19]. They compared baseline patient characteristics and analyzed their impact on disability scores after 12 months. The initial disability score was the strongest prognostic factor for continued disability in both groups, which is consistent with our findings. They also found significantly greater improvement in disability in the acute/subacute group, but in contrast to our study, the initial scores for the acute/subacute group were also significantly lower than the chronic group. Their final multivariable regression analysis also showed unemployment and higher initial levels of pain to be significant impactors on disability, which ours did not.

Psychological factors, specifically depression and anxiety, have been associated with poorer recovery of low back pain. Pincus, et al. conducted a systematic review of prospective cohort studies in low back pain to investigate psychological factors influencing the development of chronicity in low back pain [9]. They concluded that distress, depressed mood, and somatization were implicated in the transition to chronic low back pain. In a retrospective study performed by Brummett, et al. $42 \%$ of patients with low back pain had a history of anxiety or depression and were more likely to report lower physical function [8]. Pinheiro, et al. also performed a systematic review to investigate an association between depression and worse outcomes in low back pain [10]. They found 11 of 17 studies reported worse outcomes in patients with a previous diagnosis of depression. Only 2 studies found no significant correlation. Of note, our study did not find a previous mental health diagnosis to be a significant factor impacting disability score, but it was significantly correlated with higher pain scores upon discharge.

Our multivariate analysis also found that a higher BMI was associated with greater improvement in pain scores $(p=0.020)$. As mentioned above, this is in contrast to a similar study performed by Oliveira, et al. [18]. Cuesta-Vargas, et al. specifically addressed this issue in a prospective study of chronic axial low back pain patients enrolled in an 8-week course of physical therapy $(n=53)$ [11]. Subjects were divided into two groups based on BMI: a non-obese group $(\mathrm{BMI}<30)$ and an obese group (BMI > 30$)$. Subjects in the non-obese group experienced significantly greater improvement in measurements of disability, physical activity, and quality of life. However, Mangwani, et al. divided 140 patients into three groups based on $\mathrm{BMI}$ (BMI < 25, BMI 25-29.9, and BMI > 30) and found no significant difference in mean pain scores or disability scores between the groups after 6-12 weeks of physical therapy [20]. Our study would suggest that more obese patients may actually experience greater

Table 4: Comparison of Utilization of Treatments by Group.

\begin{tabular}{|c|c|c|c|c|c|c|c|c|}
\hline & $\begin{array}{l}\text { Duration of } \\
\text { Treatment } \\
\text { (weeks) }\end{array}$ & $P$ value & \# of PT visits & $P$ value & \# of Chiro visits & $P$ value & \# of Injections & $P$ value \\
\hline Duration: & & 0.479 & & 0.438 & & 0.132 & & 0.531 \\
\hline Acute/Subacute & $5.5 \pm 5.0$ & & $3.9 \pm 2.4$ & & $1.7 \pm 2.1$ & & $0.1 \pm 0.3$ & \\
\hline Chronic & $6.1 \pm 5.2$ & & $4.2 \pm 2.2$ & & $2.3 \pm 2.6$ & & $0.1 \pm 0.4$ & \\
\hline $\begin{array}{l}\text { Mental Health } \\
\text { Diagnosis }\end{array}$ & & 0.789 & & 0.645 & & 0.141 & & 0.566 \\
\hline Yes & $5.8 \pm 5.5$ & & $4.0 \pm 2.0$ & & $2.6 \pm 2.9$ & & $0.1 \pm 0.5$ & \\
\hline No & $6.0 \pm 5.1$ & & $4.1 \pm 2.3$ & & $2.0 \pm 2.3$ & & $0.1 \pm 0.4$ & \\
\hline
\end{tabular}


pain relief in a multidisciplinary spine program.

A secondary investigation of this study was to assess if there were any quantitative patterns in utilization of the various treatment options available in our multidisciplinary spine center (Table 4). We specifically compared data between the acute/subacute and chronic groups, as well as subjects with a mental health diagnosis versus those without, as these factors were significant in the multivariate analysis. We found no statistical significance in use patterns between any of the groups for any of the treatments. Some studies in the past have shown an increased utilization of health care resources in low back pain patients with a history of depression or anxiety disorder [21-23] however this was not the case in our study.

This study had several limitations. First, it was a retrospective review and therefore carries the inherent risk of bias associated with this study design. Another limitation is the use of "mental health diagnosis" to assess psychological factors that may impact low back pain recovery. Several other studies have focused on a diagnosis of depression or anxiety disorder, but we broadened our investigation also to include panic disorder and bipolar disorder based on the experience of the authors. This decision may potentially have weakened the statistical findings, as inclusion of these subjects into the "mental health diagnosis" group may have skewed the data to include more mental health diagnoses than other studies. A limitation for the secondary investigation is that the overall number of spine injections was very low for all subjects (mean 0.1 injections), and the results of this analysis likely bear very little power.

\section{Conclusion}

This study has shown that patients with axial low back pain of an acute/subacute duration showed greater improvement in disability scores and those with higher initial disability scores showed less improvement upon discharge from a multidisciplinary spine treatment center. It also showed that higher initial pain scores and a previous mental health diagnosis were significant prognostic factors for less improvement in final pain scores. The results of this study support treating both acute/subacute and chronic symptoms in a multidisciplinary spine practice model with the expectation that the acute/subacute patients may improve to a higher degree than those with chronic symptoms.

\section{Disclosures}

No disclosures and no funding was received for this study.

\section{References}

1. Andersson GB (1998) Epidemiology of low back pain. Acta
Orthop Scand Suppl 281: 28-31.

2. Mehra M, Hill K, Nicholl D, Schadrack J (2012) The burden of chronic low back pain with and without a neuropathic component: A healthcare resource use and cost analysis. J Med Econ 15: 245-252.

3. Dagenais S, Caro J, Haldeman S (2008) A systematic review of low back pain cost of illness studies in the United States and internationally. Spine J 8: 8-20.

4. Dunn KM, Croft PR (2006) The importance of symptom duration in determining prognosis. Pain 121: 126-132.

5. Valentin GH, Pilegaard MS, Vaegter HB, Rosendal M, Ørtenblad L, et al. (2016) Prognostic factors for disability and sick leave in patients with subacute non-malignant pain: A systematic review of cohort studies 6: e007616.

6. Bekkering GE, Hendriks HJ, van Tulder MW, Knol DL, Simmonds MJ, et al. (2005) Prognostic factors for low back pain in patients referred for physiotherapy: comparing outcomes and varying modeling techniques. Spine (Phila $\mathrm{Pa}$ 1976) 30: 1881-1886.

7. da C. Menezes Costa L, Maher CG, Hancock MJ, McAuley $\mathrm{JH}$, Herbert RD, et al. (2012) The prognosis of acute and persistent low-back pain: a meta-analysis. CMAJ.JAMC 184: E613-E624.

8. Brummett CM, Goesling J, Tsodikov A, Meraj TS, Wasserman RA, et al. (2013) Prevalence of the fibromyalgia phenotype in patients with spine pain presenting to a tertiary care pain clinic and the potential treatment implications. Arthritis Rheum 65: 3285-3292.

9. Pincus T, Burton AK, Vogel S, Field AP (2002) A systematic review of psychological factors as predictors of chronicity/ disability in prospective cohorts of low back pain. Spine (Phila Pa 1976) 27: E109-E120.

10. Pinheiro MB, Ferreira ML, Refshauge K, Maher CG, Ordoñana JR, et al. (2016) Symptoms of depression as a prognostic factor for low back pain: A systematic review. Spine J 16: 105-116.

11. Cuesta-Vargas Al, González-Sánchez M (2013) Obesity effect on a multimodal physiotherapy program for low back pain suffers: Patient reported outcome. J Occup Med Toxicol 8: 13.

12. Wertli MM, Held U, Campello M, Schecter Weiner S (2016) Obesity is associated with more disability at presentation and after treatment in low back pain but not in neck pain: Findings from the OIOC registry. BMC Musculoskeletal Disorders 17: 140.

13. Beurskens AJ, de Vet HC, Köke AJ (1996) Responsiveness of functional status in low back pain: A comparison of different instruments. Pain 65: 71-76.

14. Childs JD, Piva SR, Fritz JM (2005) Responsiveness of the numeric pain rating scale in patients with low back pain. Spine (Phila Pa 1976) 30: 1331-1334.

15. Fairbank JC, Couper J, Davies JB, O'Brien JP (1980) The Oswestry low back pain disability questionnaire. Physiotherapy 66: 271-273.

16. Leclaire R, Blier F, Fortin L, Proulx R (1997) A cross-sectional study comparing the Oswestry and Roland-Morris Functional Disability scales in two populations of patients with low back pain of different levels of severity. Spine (Phila Pa 1976) 22: 68-71.

17. Taylor SJ, Taylor AE, Foy MA, Fogg AJ (1999) Responsiveness of common outcome measures for patients with low back pain. Spine (Phila Pa 1976) 24: 1805-1812. 
18. Oliveira IS, Costa LOP, Garcia AN, Miyamoto GC, Cabral CMN, et al. (2018) Can demographic and anthropometric characteristics predict clinical improvement in patients with chronic non-specific low back pain? Braz J Phys Ther 22: 328-335.

19. Grotle M, Foster NE, Dunn KM, Croft P (2010) Are prognostic indicators for poor outcome different for acute and chronic low back pain consulters in primary care? Pain 151 : 790-797.

20. Mangwani J, Giles C, Mullins M, Salih T, Natali C (2010) Obesity and recovery from low back pain: A prospective study to investigate the effect of body mass index on re- covery from low back pain. Ann R Coll Surg Engl 92: 23-26.

21. Gili M, Luciano JV, Serrano MJ, Jiménez R, Bauza N, et al. (2011) Mental disorders among frequent attenders in primary care: A comparison with routine attenders. J Nerv Ment Dis 199: 744-749.

22. Konnopka A, Schaefert R, Heinrich S, Kaufmann C, Luppa M, et al. (2012) Economics of medically unexplained symptoms: A systematic review of the literature. Psychother Psychosom 81: 265-275.

23. Stewart WF, Yan X, Boscarino JA, Maeng DD, Mardekian $J$, et al. (2015) Patterns of health care utilization for low back pain. Journal of Pain Research 8: 523-535. 